\title{
Effect of Including Detector Response in SPECT Quantification of Focal I-131 Therapy
}

\author{
Kenneth F. Koral, Qiang Lin, Adil Akhtar, Jia Li, Yuni K. Dewaraja and Jeffrey A. Fessler
}

\begin{abstract}
With a regularized strip-integral (1D) SAGE reconstruction, circular-orbit SPECT estimates of phantom focal 131-I activity vary with changes in the level of uniform background. They also vary with changes in image resolution due to different settings of the radius of rotation. To solve these problems, we investigated the effect of employing two different depth-dependent detector-response models. A regularized plane-by-plane (2D) SAGE algorithm reduced dependence of the counts-to-activity conversion factor on relative background concentration by $37 \%$ compared to the 1D SAGE. With unregularized multi-plane (3D) OSEM reconstruction, initial results showed: 1) a conversion factor that was independent of relative background concentration, and 2) a recovery coefficient that was approximately 1 for any sphere volume down to $20 \mathrm{cc}$. We conclude that using a 3D detector-response model has the potential to eliminate bias problems. For a patient, the preliminary activityestimate changes using 3D OSEM compared to 1D SAGE were: 1 ) $+16 \%$ for a large tumor, and 2) $-35 \%$ for a small tumor for which recovery-coefficient-based-correction-factor errors can be large.
\end{abstract}

\section{INTRODUCTION}

Kessler et al explicitly pointed out that, in the general case, each pixel of a reconstructed image suffers from both spill out of activity that should be there and spill in of activity from other pixel sources. Both effects are due to finite resolution. For quantification of activity in a volume of interest ( $\mathrm{VoI})$, they suggested the use of phantom-based hot-spot and cold-spot recovery coefficients to correct for these distortions [1]. Others have pointed out problems with that method, however [2].

When the outlines of specific VoI were known from a CT-SPECT image fusion by means of sequential scanning with a multimodality imager, a group at the University of California at San Francisco (UCSF) has used a template method for activity quantification. With one version of the template method, first a subtraction accounts for spill in to the target from each background object, and then a division of the difference by a value less than one accounts for spill out from the target [3]. The initial reconstruction gives the ratio of the relative activity of each background object compared to the target activity. This ratio is required for the

Kenneth F. Koral, is with the University of Michigan Medical Center, Ann Arbor, MI 48109-0552 USA. (telephone: 734-764-5103, e-mail: kenkoral@umich.edu).

$\mathrm{Jia} \mathrm{Li}$ is with the Department of Electrical Engineering and Computer Science, the University of Michigan, Ann Arbor, MI 48109-2122 USA (telephone: 734-647-8389, e-mail: jiali@engin.umich.edu). processing. Since the initial reconstruction is tarnished by finite resolution, one can expect some inaccuracy in the ratio and therefore in the resultant quantification.

As the UCSF group has done, we also have relied on the outline of specific VoI from a CT-SPECT image fusion. However, we have used a sphere-in-a-cylinder activity calibration and have up until now employed a conversion factor (reconstructed counts/MBq), $\mathbf{C F}$, that varies both with the radius of rotation, $\mathbf{R}$, of the circular orbit of the SPECT acquisition, and also with a measured concentration-ratio parameter, $\mathbf{b}$, that equals the concentration $\left(\mathrm{MBq} / \mathrm{cm}^{3}\right)$ of the background divided by the concentration of the target [4]. This ratio parameter monitors the relative magnitude of count gain due to spill in from the background activity compared to count loss due to spill out from the target. Since no explicit subtraction for the effects of spill in of counts is needed, noise-amplification is avoided. We have made measurements of tumor activity in lymphoma patients being treated with tositumomab using this calibration (4). For those measurements, we employed circular-orbit SPECT with a high-energy general all-purpose (HEGAP) collimator. Reconstruction was carried out with regularized strip integral 1-D SAGE (5). Here, we both employ an ultra-high-energy parallel-hole (UHEGAP) collimator to reduce septal penetration and improve contrast (6), and also investigate the effect of employing two different reconstruction algorithms with depth-dependent detector response.

\section{METHODS}

To calibrate the UHEGAP collimator, SPECT images of a $200 \mathrm{~cm}^{3}$, known-I-131-activity sphere situated off axis in an elliptical water phantom were acquired using the UHEGAP collimator, a circular orbit, and a Picker Prism camera. Uniform-background level was varied (4 concentration-ratio values, nominally $0,1 / 8,1 / 4$ and $1 / 2$ ) as well as radius of rotation (5 values, 19, 21, 23, 24.5 and $26 \mathrm{~cm}$ ) to produce 20 separate acquisitions in total. To determine FWHM parameters for the 2D reconstruction, we used Monte Carlo simulation. It generated planar images of a point source at several distances from the face of the collimator. Spatial averaging was enabled to minimize the collimator hole pattern. For the FWHM parameters for the 3D reconstruction, we employed new experimental data of the same type. The experimental point source data were fit by a program that estimated a center location and the parameters of a 2D Gaussian.

Monte-Carlo simulation also generated SPECT data to produce recovery coefficients for spherical volumes different than $200 \mathrm{~cm}^{3}$. Four different values of uniform background were investigated but it was assumed that $\mathbf{R}$ would have no 
effect on the recovery coefficients. Up until now, we have corrected the activity for a tumor target volume that is not equal to $200 \mathrm{~cm}^{3}$ by the inverse of the recovery coefficient (RC) appropriate for the spherical volume equal to the tumor volume. The correction is greater than 1 for volumes smaller than $200 \mathrm{~cm}^{3}$, and less than 1 for greater volumes.

Attenuation-correction-included reconstruction using 20 iterations was carried out with 1) 1D SAGE, 2) regularized plane-by-plane 2D depth-dependent-detector-response SAGE, or 3) unregularized 3D table-driven depth-dependent-detectorresponse OSEM. For 3D reconstruction, OSEM is more practical (less computing time and memory) than SAGE would have been. Regularization was disabled in the 3D case to maximize resolution. In all cases, 1) the attenuation map was derived from energy extrapolation of the fused CT image, and 2) scatter correction was carried out during reconstruction by using a pixel-by-pixel estimate from a triple-energy-window technique. The measured value for $\mathbf{b}$ was calculated from the number of counts within the VoI of the target (sphere or tumor), the number of pixels in that VoI, the number of counts within the VoI of the object (ellipse or abdomen), and the number of pixels in that VoI. For the activity concentration in the target, the number of counts was divided by the number of pixels. For the background activity concentration, the number of target counts was subtracted from the net counts within the object and the target number of pixels was subtracted from the net pixels within the object. The difference in counts was then divided by the difference in pixels to yield the background concentration.

A lymphoma patient (ID\# 76) was imaged with SPECT with $\mathbf{R}_{\mathbf{0}}=24.63 \mathrm{~cm}$ at 44 hours after a therapy administration of $4.04 \mathrm{GBq}$ of 131-I tositumomab. For the 1D and 2D reconstructions, bilinear interpolation was used to choose $\mathbf{C F}$ from the four calibration curves nearest to $\mathbf{R}_{\mathbf{0}}$ and the measured $\mathbf{b}_{\mathbf{0}}$ for each tumor. The recovery-coefficient-based correction value for the volume equal to the measured volume of the tumor was interpolated from the two curves nearest to the measured $\mathbf{b}_{\mathbf{0}}$ value for each tumor and applied. The correction value multiplied the initial activity estimate to produce the final estimate. For the $3 \mathrm{D}$ reconstruction, the average of 4 measured values of $\mathbf{C F}$ was employed, and no recovery-coefficient correction was applied to either of the two tumors.

\section{RESUlts}

With 1D SAGE reconstruction, CF exhibited a linear dependence on measured $\mathbf{b}$ for each of the 5 values of $\mathbf{R}$ (average $r^{2}=0.992$ ). A sample dependence is shown by the least-squares-fit line at the bottom of Figure 1. Although not shown, at a fixed $\mathbf{b}, \mathbf{C F}$ was also linear with $\mathbf{R}$. The results with 1D SAGE for the recovery-coefficient-based correction factor in the case of no background are shown starting at the upper left of Figure 2. The correction had a similar dependence on volume as that with a HEGAP collimator, but the values were closer to unity.

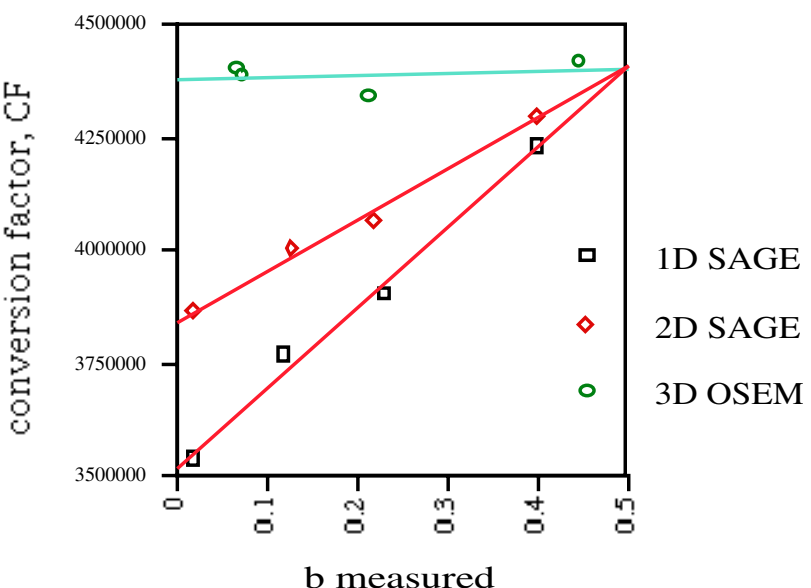

Figure 1. Reduction in slope of calibration curve with detector response included. Counts-to-activity conversion factor $\mathbf{C F}$ is plotted versus measured activity-concentration ratio (background over sphere) b. SPECT radius of rotation $\mathbf{R}$ equals $23 \mathrm{~cm}$. The dependence is reduced with $2 \mathrm{D}$ SAGE compared to 1D SAGE and essentially disappears with the 3D system model.

For unregularized plane-by-plane 2D depth-dependentdetector-response SAGE reconstruction, the Monte-Carlo point-source data yielded a linear dependence: FWHM = $8.96 \mathrm{~mm}+0.067 *$ distance. With 2D SAGE, CF again varied linearly with measured $\mathbf{b}$ (average $r^{2}=0.969$ ). A sample dependence is shown by the middle line of Figure 1. The slope of the fitted line was less with the 2D reconstruction than with the $1 \mathrm{D}$ due to less spill into the sphere VoI of counts from non-zero background. The intercept conversion factor was greater, due to less spill out of counts from the sphere $\mathrm{VoI}$ at $\mathbf{b}=0$. Averaged over the 5 $\mathbf{R}$ values, 1) the slope was reduced $37 \%$, and 2) the intercept was increased $9.6 \%$. Although not shown, at a fixed background, $\mathbf{C F}$ was again linear with $\mathbf{R}$, but with a smaller slope than with the $1 \mathrm{D}$ reconstruction. With 2D SAGE the recovery-coefficient-based-correction-factor values were considerably reduced compared to those with 1D SAGE (see figure 2). There were similar reductions at other values of $\mathbf{b}$.

For unregularized 3D table-driven depth-dependentdetector-response OSEM reconstruction, the FWHM values were similar to those from Monte Carlo but the value for the smallest distance was unusually small. Therefore, straightline interpolation of the FWHM between depth points was used to construct the table rather than a global linear fit. With 3D reconstruction, initial results (i.e., those with $\mathbf{R}=23 \mathrm{~cm}$ ) showed that the counts-to-activity conversion factor was nearly independent of background (see top curve of Figure 1). The initial results $(\mathbf{b}=0)$ also showed a recovery coefficient that was approximately 1 for any volume down to $20 \mathrm{cc}$ (see bottom curve of Figure 2). 

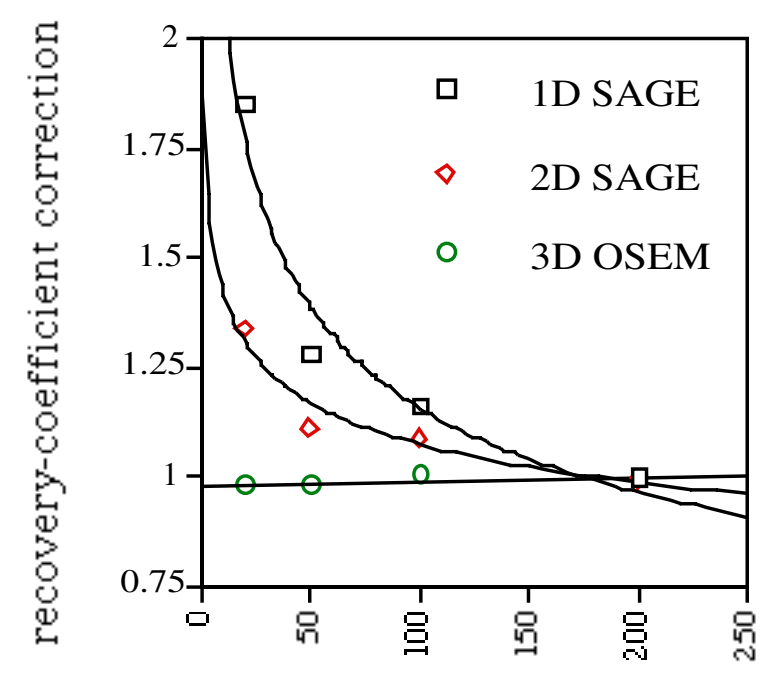

Volume cc

Figure 2. Recovery-coefficient-based correction factor plotted versus sphere volume. The nominal background concentration ratio is zero. The $1 \mathrm{D}$ and $2 \mathrm{D}$ SAGE results are fit with a power function, the 3D OSEM results with a straight line. There is the least dependence on volume with 3D OSEM.

For the patient, the activity estimates from the various reconstructions with and without recovery-coefficient correction are shown in Table 1. We preliminarily assumed that with 3D OSEM 1) the conversion factor is independent of $\mathbf{b}$ for all radii, and 2) there is no need for a correction for volume. For the large tumor the activity estimate was increased by $10 \%$ (2D versus 1D) and by $16 \%$ (3D versus 1D). Perhaps more importantly, for a small tumor the 3D estimate was $35 \%$ less than the 1D estimate. Note that the accuracy of the 1D estimate for the small tumor rested heavily on the correctness of the recovery-based correction factor. Error in this factor could make the estimate too large, explaining the $35 \%$ decrease. The large changes in activity estimates from 3D reconstruction could easily affect the accuracy of the prediction of response by tumor dosimetry.

Figure 3 shows a slice that passes through two prongs of the 1231 cubic $\mathrm{cm}$ tumor for the patient. This large tumor is seen to have much less uniform uptake of the radioiodine with the unregularized 3D OSEM reconstruction.

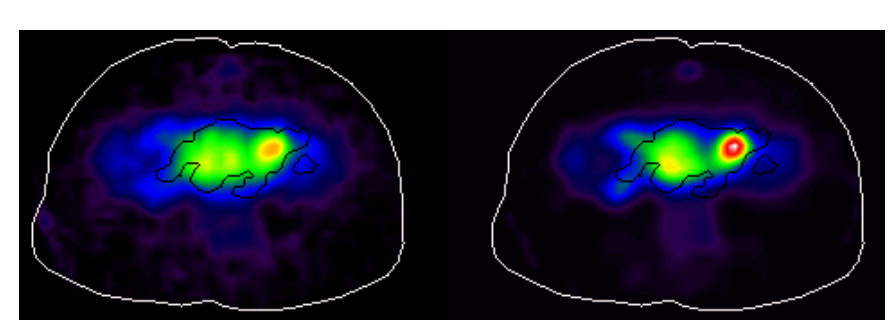

Figure 3. One slice of the patient image set. Body outline is shown in white. Outline of tumor regions is shown in black. Left: result with regularized 1D strip-integral SAGE. Right: 3D OSEM.

For the same patient, figure 4 shows two other, more superior slices that are separated from each other by $8 \mathrm{~cm}$. With 3D OSEM (right), the uptake in the spleen and in both kidneys is stronger than with 1D SAGE. On the other hand, the liver uptake and that in the aorta is weaker.

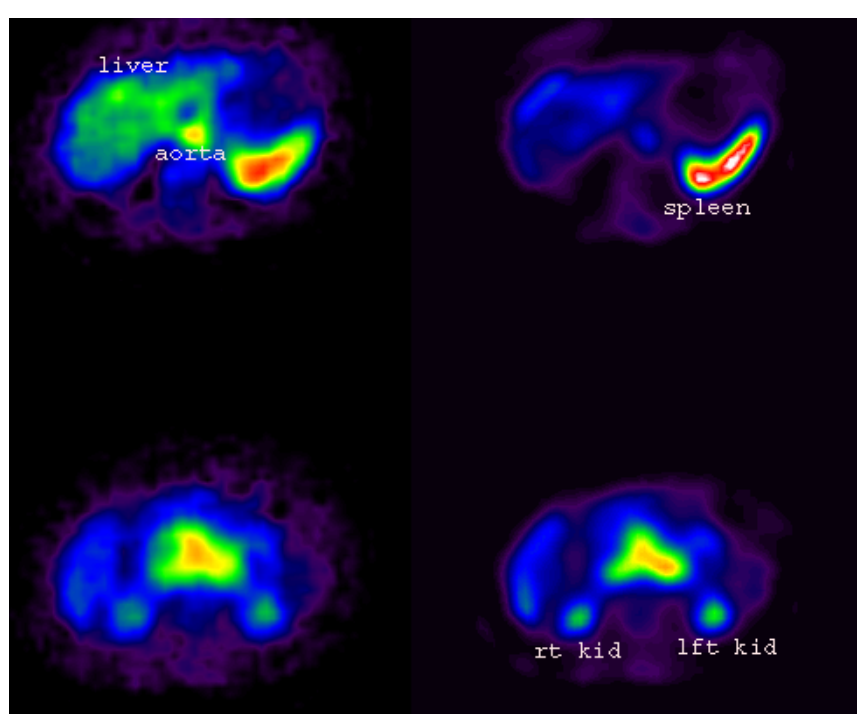

Figure 4. Two more slices from the patient image set. These are located superiorly from that of Figure 3. Left: result with regularized 1D strip-integral SAGE. Right: 3D OSEM.

Table 1. Effect of including detector response (2D or 3D) in reconstruction on the activity estimates for two tumors in a lymphoma patient. Cor. $=$ correction.

\begin{tabular}{|c|c|c|c|c|c|c|c|c|c|}
\hline \multirow{3}{*}{$\begin{array}{c}\text { Tumor } \\
\text { volume } \\
\text { (cubic } \\
\mathrm{cm} \text { ) }\end{array}$} & \multicolumn{6}{|c|}{ Activity in microcuries } & \multirow{2}{*}{\multicolumn{3}{|c|}{$\begin{array}{c}\text { With RC cor., difference } \\
\text { between algorithms* }\end{array}$}} \\
\hline & \multicolumn{3}{|c|}{ No RC-based correction } & \multicolumn{3}{|c|}{ With RC-based correction } & & & \\
\hline & $1 \mathrm{D}$ & $2 \mathrm{D}$ & $3 \mathrm{D}$ & $1 \mathrm{D}$ & $2 \mathrm{D}$ & $3 D^{7}$ & 2Dvs1D & 3Dvs2D & 3Dvs1D \\
\hline 2.1 & 0.20 & 0.25 & 0.20 & 0.31 & 0.31 & 0.20 & 0.0 & -35.4 & -35.4 \\
\hline 1231 & 1722 & 1696 & 1539 & 1324 & 1452 & 1539 & +9.7 & +6.0 & +16.3 \\
\hline
\end{tabular}

$*$ Difference $($ in percent $)=\left\{\left(\mathrm{A}_{\text {new }}-\mathrm{A}_{\text {base }}\right) / \mathrm{A}_{\text {base }}\right\} * 100 \%$

${ }^{7}$ For $3 \mathrm{D}$, a correction factor of 1 , or no correction, has been applied 


\section{DISCUSSION AND CONCLUSION}

For our usual quantification method to have less bias, we would like it to be less dependent on the parameters that affect the activity. That is, the $\mathbf{R}$ value should usually be accurately known for a patient, but the tumor location within the body may be different than that of the calibration sphere within the elliptical-cylinder phantom. Also, the appropriateness of the measured $\mathbf{b}$ value is questionable because the background activity is distributed non-uniformly for a patient while it was distributed uniformly for the calibration phantom. Similarly, the dependence of the true total-activity recovery coefficient on volume may be different in the patient case (non-spherical tumor shape) than in the phantom case. Finally, the measured volume of a tumor may be different than the true value, which would lead to error in the activity estimate when using a volume-dependent recovery coefficient. The present results show that reconstruction with a 3D depth-dependent system-response model has the potential to eliminate the above bias problems when we have data acquired with the UHEGAP collimator.

\section{ACKNOWLEDGMENT}

This work was supported by Grants R01 CA87955 and CA80927 awarded by the National Cancer Institute, United States Department of Health and Human Services. Its contents are solely the responsibility of the authors and do not necessarily represent the official views of the National Cancer Institute.

\section{REFERENCES}

1. Kessler RM, Ellis JR, Eden M. Analyses of emission tomographic scan data: limitations imposed by resolution and background. J Comput Assist Tomogr, 1984;8:514-522.

2. Geworski L, Knoop B, de Cabrejas M, et al. Recovery correction for quantitation in emission tomography: a feasibility study. Eur J Nucl Med, 2000;27:161-169.

3. Tang HR, Da Silva AJ, Matthay KK, Price DC, Huberty JP, Hawkins RA, Hasegawa BH. Neuroblastoma imaging using a combined CT scannerscintillation camera and 131I-MIBG. J Nucl Med, 2001;42:237-247.

4. Koral KF, Dewaraja Y, Li J, Barrett CL, Regan DD, Zasadny KR, Rommelfanger SG, Francis IR, Kaminski MS, Wahl RL. Initial results for Hybrid SPECTconjugate-view tumor dosimetry in 131I-anti-B1 antibody therapy of previously untreated patients with lymphoma J Nucl Med, 2000;41 1579-1586.

5. Fessler, J. A. and Hero, A. O. Penalized maximumlikelihood image reconstruction using space-alternating generalized EM algorithms. IEEE Tr. Im. Proc., 1995;4(10):1417-1429.

6. Dewaraja YK, Ljungberg M, Koral KF. Accuracy of 131I tumor quantification in radioimmunotherapy using SPECT imaging with an ultra-high-energy collimator: Monte Carlo study 2000;J Nucl Med, 41 1760-1767. 